\title{
BIOMEDICAL RESISTANCE TO ETHNOMEDICINE IN BOTSWANA*
}

\author{
EVELYN L. BARBEE \\ The University of Michigan, 400 N. Ingalls Street, Ann Arbor, MI 48109, U.S.A.
}

\begin{abstract}
The issue of whether and how ethnomedical practitioners can be incorporated into existing biomedical systems in developing countries continues to be debated. Although a number of reasons have been advanced as to the barriers to incorporation, none of these take into account the views of nurses on incorporating ethnomedical practitioners into the biomedical system. Data from Botswana, where the largest group of biomedical personnel are nurses are presented to show the reasons why nurses are opposed to collaboration with the two groups of ethnomedical practitioners, spiritual healers and traditional healers. Because they are socialized to two belief systems about health and healing, and because of their position in the biomedical health system, the nurses are caught in a dialectical tension between their traditional beliefs and their acquired beliefs. They refuse to collaborate with traditional healers because they believe that traditional healers practice sorcery. They refuse to collaborate with spiritual healers because they do not view them as health care providers.
\end{abstract}

\section{INTRODUCTION}

In recent years concern about the delivery of health services in developing nations has led to a reaffirmation of the role of traditional specialists as health care providers. This re-affirmation of the traditional specialists role became officially sanctioned at the World Health Organization's AlmaAlta Conference on Primary Care in 1978 [1]. Since the thrust of Primary Care, as Defined by WHO [1] stresses a community based approach, WHO made a number of recommendations in regard to the possible use of traditional specialists in Primary Care [2]. Thus, one Report included the recommendations that (1) biomedical personnel and students should be acquainted with the principles of traditional medicine and (2) that traditional healers should be retrained for appropriate use in primary care [3]. The first recommendation assumes that biomedical students and personnel, in a given developing country, are not acquainted with the principles of traditional medicine in their country. The second recommendation suggests that traditional specialists want re-training, that they want to be a part of the biomedical system, and that retraining them for use in biomedicine will make traditional specialists more acceptable to biomedical practitioners. Despite the sanctions of WHO and other funding agencies, the number of programs in developing countries that incorporate traditional specialists into the biomedical system remains small [4].

A number of reasons have been advanced in an effort to understand the problems inherent in linking traditional specialists with the biomedical system. Neumann and Lauro [5] note that a major complication to cooperation between ethnomedical and biomedical systems is caused by plural medical systems. Ethnomedicine refers to that domain of indigenous medicine which is not precisely derived from the conceptual framework of biomedicine [6]. Eth-

\footnotetext{
* Research on which this paper is based was supported by the African American Scholars Council.
}

nomedical systems like biomedical systems are "a patterned interrelated body of values and deliberate practices governed by a single paradigm of meaning, identification, prevention, and treatment of illness and disease" [7]. Ethnomedical and biomedical systems may be mutually influencing, however their basic values are part of the socio-cultural context in which they originate. As a result, identifying and determining which ethnomedical practitioners should be involved in Primary Care is a problem. Pillsbury [4] cites structural reasons, e.g. lack of implementation of national policy, few evaluation studies on the efficacy of incorporation and the differing priorities of key decision makers as sources of resistance to the linking of ethnomedical and biomedical practitioners. Yoder [8], in stressing that cooperation between biomedical and ethnomedical should work both ways, argues that cooperation must be proceeded by each group gaining knowledge and experience in the practice of the other.

This paper discusses some of the socio-cultural issues that must be recognized before cooperation can occur among practitioners in Botswana in general and more specifically in the Serowe area of Botswana. A primary emphasis is on the nurses' view of collaboration with traditional specialists and how their beliefs about ethnomedical practitioners affects their willingness to collaborate. It is argued that nurses, the pivotal group of biomedical practitioners in Botswana, must be involved in any program that has the goal of collaboration among biomedical and ethnomedical practitioners.

In order to better understand the nurses' position on collaboration with ethnomedical practitioners, it is necessary to know more about the baTswana, their biomedical care system, and the primary roles of their ethnomedical practitioners. The data in this paper are derived from anthropological fieldwork in Botswana from 1975 to 1976. Methods of data collection included participant observation, and formal and informal interviews with nurse, traditional and spiritual healer informants. The principle sites of data collection were Gaberone, the capital of Botswana, 
Serowe, and the surrounding areas of the Central District. Additional data were collected from the villages of Ramotswa and Molepolole.

The Republic of Botswana is located in southern Africa. Its total area of approx. 223,000 square miles makes it about the size of the state of Texas. Completely landlocked, it is bordered on the west by Namibia, on the south and southeast by South Africa, and the northeast by Zimbabwe, and on the north by the Caprivi Strip. Geographically, Botswana is part of the southern Central African Plateau and is 3300 feet above sea level. In the central and southwestern portions of the country, the plateau forms a shallow basin that is occupied by the Khalahari Desert. A semi-arid region, the Khalahari comprises approx. two-thirds of the country. The climate in Botswana is generally sub-tropical. Rainfall tends to be seasonal, low, and erratic. Since the rainfall is highest, the soil more productive, and the grazing land better in the eastern part of the country, the bulk of the population lives here.

The baTswana are pastoralists and agriculturalists. Although generally regarded as a nation of cattle raisers, cattle wealth is unevenly distributed and a large part of the population is poor. The majority of the baTswana live in rural villages some of which are quite large. Most of the principle villages, where the chiefs have their headquarters are quite large. The custom of living in large villages has resulted in the baTswana adoption of a system of cattle posts and agricultural lands that are appendages to but great distances from, the village. The two principle languages spoked in Botswana are English and seTswana.

The baTswana are classified as one of the three major divisions of the Sotho group in southern Africa and are sometimes referred to as Western Sotho. Although baTswana are located in various parts of South Africa, for example, Rustenberg and Mafeking, this paper is concerned with the baTswana who live in Botswana and more specifically with those who are identified as baNgwato. In Botswana there are eight principle ethnic groups [9]. Each of these groups has a population of mixed origins. The most extreme of which is the baNgwato of whom more than five-sixths are derived from other ethnic groups [10]. The baNgwato are primarily found in the Central District. Serowe, the capital of the Central District is located in the northeastern section of Botswana. Serowe, with its population of approx. 25,000 , is divided into wards and sub-wards for administrative purposes. The most significant social units of the baNgwato are the family group which is organized agnatically and the household. The core members of a household generally include a man, his wife, and their unmarried children. The family group live in homesteads that are grouped together in a sub-ward.

\section{BIOMEDICAL HEALTH CARE}

The biomedical health care system in Botswana is geared to meet the needs of the country's rural based population. In the village of Serowe and the surrounding areas of the Central District, biomedical care is provided by both the central government and the Central District Council. The system operates on the premise of hierarchical referral. At the top of the hierarchy are the hospitals. The hospital in Serowe operates under the auspices of the central government. Like all hospitals in Botswana, it serves as a referral center for patients from other biomedical facilities. In addition to providing curative care, the hospital provides preventive care through its ante and post natal clinics and tertiary care through its tuberculosis clinic. Next on the referral hierarchy are health centers. Because they are generally located in rural areas far from hospitals, health centers have beds for both maternity and general patients. Better equipped than clinics, the health center offers both curative and preventive care. On the next level of biomedical care are the clinics. Clinics offer outpatient services, preventive care, and most have beds for maternity paticnts. Most clinics are in the rural areas. The lowest level in the biomedical hierarchy is occupied by health posts. Health posts are equipped to provide a minimum level of curative care, e.g. cough syrups and aspirin.

\section{BIOMEDICAL PERSONNEL}

The three major cadres of biomedical personnel are physicians, nurses and Family Welfare Educators. At the time of this study there were approx. 65 physicians, over 400 nurses, and an estimated 60 Family Welfare Educators in Botswana. Approximately 50 of the physicians worked in government hospitals. Ninety percent of the physicians employed in the government hospitals were expatriates. The largest number of biomedical personnel in Botswana are nurses. The overwhelming majority of the nurses are women, and with the exception of a few expatriate volunteers, they are all either baTswana or African recruits who speak seTswana. Nurses are frequently referred to as the 'backbone' of biomedical care. Two levels of nurses are prepared in Botswana, enrolled nurses (ERNs), similar to licensed practical/ vocational nurses in the United States and registered nurses. Enrolled nurse programs are 2 years in length and cover the same areas as training programs for registered nurses. The basic differences between the two are that enrolled nurses do not receive as much depth training as registered nurses and are trained to work under the supervision of a registered nurse. Enrolled nurses who receive a merit pass in their post-training examinations, have the opportunity to take a two year midwifery course. Enrolled nurse programs are operated by the Ministry of Health in four of the government hospitals.

The other level of nurses prepared in Botswana is registered nurses. Registered nurses are prepared at the National Health Institute in Gaberone. The registered nurse program is 3 years in length and stresses maternal and child health, public health, and mental health. After completion of the program, all registered nurses automatically proceed to midwifery. All registered nurses in Botswana are trained as midwives because they deliver babies in the rural biomedical facilities. Unlike other countries, the baTswana do not use traditional birth attendants [11]. In addition to the program that prepares registered nurses, there is a 3-year program at the University of Botswana 
that prepares registered nurses to become tutors of nursing [12].

The bulk of the nurses in Botswana work for either the central government or the District Councils in the hospitals and rural biomedical care facilities. These rural facilities are staffed exclusively by nurses and auxiliary personnel. In the rural clinics and health centers, on their initial visit patients are seen by a nurse who takes a history of their complaints. Most of the patients are diagnosed and treated by the nurses. Those patients who do not respond to treatment are referred to the physician who visits fortnightly. Patients with difficult complaints, for example, urinary retention, congenital deformities and advanced cases of tuberculosis are referred to the hospital by either the nurse or the physician.

In addition to seeing patients, nurses also deliver babies and conduct the family planning, child welfare and antenatal clinics. Their duties in the child welfare clinic include weighing children, advising mothers on diet and hygiene and giving immunizations. In the antenatal clinic they weigh the prospective mothers, take their blood pressure, test their urine, record the height of the uterus and listen to fetal heart tones. Nurses also visit the surrounding villages and give immunizations.

There are a number of problems associated with working in the rural clinics. Transportation is erratic at best. In addition there is a lack of equipment and supplies. Although the nurses lack the large number of diagnostic instruments that characterize biomedicine in the United States, they meet two of the most critical conditions needed to make a medical diagnosis. First, they share the same sociocultural system as their patients. Thus they are able to ascertain whether their patients have been seen by an ethnomedical practitioner. Second, because they are the same sociocultural system as their patients, communication is greatly enhanced. As a result, the nurses are able to get more detailed and more accurate health histories than expatriate physicians. These histories provide the bases for diagnoses.

Family Welfare Educators are trained at Sebele Training College near Gaberone. These workers are recruited from their villages by the District Councils. The requirements to be a FWE are: woman, over the age of 20 with at least one child, ability to speak English, and completion of the seventh grade in school. In a 3 month training program, FWEs take basic subjects in first aid, nutrition, family planning, health education, public health (e.g. home visits), diagnosis of sample ailments (e.g. scabies) and community development and social problems. The work relationship between the FWEs and the nurses is a close one. In their work, FWEs refer patients to the nurse. In Botswana, nurses and FWEs are the persons who carry the health message to the people.

\section{ETHNOMEDICAL PRACTITIONERS}

It is important to note that, traditionally, the baTsuana do not make the separation between religion and healing that is usually made in biomedicine. The overall religious belief system of the baTsuana and the function of this belief system in their lives, will not be discussed in this paper.
The ethnomedical system that coexists with biomedicine in Botswana is comprised of two major groups, traditional specialists (dingaka) and spiritual healers (dingaka tsa metse). The largest group of traditional specialists are diviners. Diviners diagnose through bonecasting and use various herbs in their treatments. Although bones (diatola) are used by diviners for a variety of purposes, in regard to healing, diviners in Botswana use their skills for three major purposes. These purposes are: to determine what is wrong with an individual; to decide whether they can treat an individual or whether the person should be referred to a hospital; and to place an individual's symptoms within a social context, that is, to determine the causc of an illness.

In the Serowe area, traditional specialists are men and women who learned their skills from serving an apprenticeship with another healer. Most have been working as independent practitioners for 20 years or more and are $\mathbf{5 0}$ years of age or older. Social and cultural transformations in baTswana society have created a number of problems for the traditional specialists. The combination of general lack of apprentices, people not paying their fees, and the competition from spiritual healers, have caused the traditional specialists concern about the fate of traditional medicine (bongaka) in Botswana. For the most part traditional medicine is surrounded by secrecy. A major reason for the secrecy surrounding traditional medicine is the pervasive baTswana belief in the practice of sorcery. Belief in the practice of sourcery is closely connected with the diviner's use of bones and herbs.

The baTswana believe in the practice of sorcery [13]. This is not to imply that the baTswana are obsessed with sorcery. Within the context of the baTswana society, sorcery is a danger that must be faced almost daily [14]. Essentially, the baTswana believe that precautions can be taken that are protective against sorcery. As Schapera [14] notes, the baTswana believe that people practice sorcery and that those who practice it are well aware of what they are doing. Furthermore, they believe that any person, male or female might practice sorcery. In the past, sorcerers and their victims were closely related; sorcery was usually employed in settings of domestic conflict, and the major motive was jealousy. Today, although the major motive for the practice of sorcery remains jealousy, the network for sorcery accusations has extended to include non-kin. The baTswana belief in the practice of sorcery primarily revolves around a condition known as sejeso, or 'Tswana poisoning' This poisoning consists of putting some lethal substance in a person's food or drink. The food or drink is then given to the person to be ensorcelled. The baTswana connect diviners to sorcery in three ways. First, they believe that diviners can be hired to impose sorcery on an intended victim. Second, they believe that the herbs used in sorcery can be purchased from a diviner. Third, the diviners use the bones to determine whether a person has been ensorcelled. Although diviners deny practising sorcery, a number of them say that they can determine whether a person has been ensorcelled and by whom from the bones. However, the baTswana reason that anyone who can diagnose sorcery can also perform it. 
Spirituai healers are a relatively recent group of ethnomedical practitioners. Historically, spiritual healing in Serowe evolved from African Independent Churches of the Zionist and Ethiopian types in South Africa [15]. One important fact about spiritual healers is that they are not independent practitioners. All of them are associated with African Independent churches. Although some healers see patients in their homes, they essentially do their healing under the aegis of some church. In Serowe, the church leaders have syncretized baTswana purification rites and taboos with the Christian ritual and emphasize faith healing. In the Serowe area there are two types of spiritual healers. The first type use prayer only in their healing. The second, far more numerous type, use prayer in combination with water and ashes. Unlike the diviners, very few of the spiritual healers are trained for their positions. Most became healers as the result of some former experience that 'called' them into spiritual healing. Most of these experiences were illness experiences, that the healers referred to as 'mental illnesses'.

In their diagnoses of illness, spiritual healers depend primarily upon an elicitation of the patient's symptoms, prayer and opening the Bible. Basic to the treatment approach of spiritual healers is the use of blessed water and prayer. As an adjunct to the use of water, many healers add ashes to the water. The majority use sewasho, the ash of cow dung. Several healers say that they use cow dung because cattle eat all types of herbs and grasses.

With two exceptions, both traditional specialists and spiritual healers treat the same symptoms in both adults and children. Spiritual healers specialize in treating mental disorders, whereas traditional specialists specialize in treating the cause and effects of sorcery. Spiritual healers are most often found in large villages and urban areas because most of their churches are in large population areas. Traditional specialists, like the biomedical system, are more widely dispersed. As component groups of the ethnomedical system, traditional specialists and spiritual healers do not cooperate with each other. The basic relationship between the two groups is one of competitive antagonism.

\section{PATTERNS OF UTILIZATION}

Unlike the peoples of many African countries [16-18] baTswana adults do not use herbal remedies for the treatment of common illnesses. When the baTswana suffer a common illness they are quick to use patent medicines. In terms of healing, the baTs wana recognize the existence of biomedicine, traditional specialists, and spiritual healers and they choose differentially from among these three categories. Each of the three groups of practitioners is viewed by health care consumers as having a particular area of expertise. Thus, trauma, accidents, pregnancy and birthing are exclusively seen in the biomedical system, and more specifically by nurses. Mental illness conditions are seen more by spiritual healers. The major role of the traditional specialists lies with treating the cause and effects of sorcery. Collectively, the baTswana believe that all of the groups are competent in treating the same illnesses.
However, they do not believe that all three are equally competent to treat all illnesses. In terms of chosing either the biomedical or the ethnomedical system, the baTswana base their choice upon their particular illness, the reputation of the practitioner. and their faith that a practitioner can cure their illness. Faith in the efficacy of a particular practitioner requires that the patient not take medications from any of the two systems concomitantly because the medicines from one would negate the effects of the other. Furthermore, those who use spiritual healers do not go from them to traditional specialists. However, patients who have used traditional specialists feel free to use spiritual healers. Moreover, those who use the ethnomedical system also use the biomedical system.

\section{DISCUSSION}

The structure of the biomedical care system in Botswana, with its emphasis on hierarchal referral. places nurses at the critical juncture in the referral network. In the rural biomedical facilities nurses initiate all referrals to the physician and many referrals to the hospital. Nurses are also at the critical juncture in the hospitals, for it is they who interpret patient complaints and needs to the expatriate physicians. The majority of nurses in Botswana are baTswana. Those who are not, are seTswana speaking African from South Africa. The nurses hold beliefs about health and illness from two domains, that of their culture and that of western biomedicine. This dual belief system, coupled with the nurses presence at the critical juncture in the biomedical referral network, places the nurses in the role of primary 'broker' between traditional baTswana culture and biomedicine.

The position of 'brokers' is an exposed one because brokers must serve some of the interests of groups operating on both the community and national levels [19]. In this exposed position the brokers must cope with the conflicts raised by the collision of these interests. In the rural areas nurses are community based members of the biomedical system. As a result they mediate between the community and the biomedical system.

In their formal and informal dealings with the people, nurses share the same baTswana cosmology about health and illness as do the people in the community. At the same time they also hold the biomedical beliefs about health and illness. When the two come into conflict, it is the nurses who act as buffers between the two systems. For example, all the nurses believe that it is a waste of time for patients to seek care from traditional specialists and spiritual healers before seeking biomedical care. However, they also say that denigrating patients for seeking care from the ethnomedical system serves no useful purpose for the biomedical system. In their position as brokers, nurses in Botswana appear to have a greater investment in biomedical beliefs about health and illness than those of baTswana culture. This investment is exemplified by the nature of their complaints. The nurses' complaints concern lack of staff, lack of equipment and supplies, and obstacles 
to independent practice. The overall focus of their concerns is with factors that they feel interfere with the delivery of health care. This same investment in biomedical beliefs about health and illness affects their views about cooperation with traditional specialists and spiritual healers.

The nurses are essentially ensnared in a dialectic between traditional and biomedical beliefs [20]. The tensions created by being involved in this dialectic serve to make the nurses extremely ambivalent about cooperating with traditional specialists. Comaroff [20] identifies three ideological positions available to baTswana in South Africa caught in the dialectic between their culture and the wider world. These three positions are: (1) reject baTswana culture and identify with Western culture; (2) assert the primacy of baTswana culture and only adopt useful Western concepts; (3) pursue assimilation of baTswana and western ideology. As an example of the third alternative, Comaroff [20] gives the syncretism which is exemplified by the Zionist sects.

The nurses mediate the basic contradictions created by the dialectical tensions between their traditional and biomedical beliefs in two ways. A small number have chosen to reject their culture in favor of the Western concepts of health and illness. These nurses do not believe that they should cooperate with traditional specialists. The majority of the nurses have adopted a position of peaceful coexistence that is based upon the empirical nature of baTswana society and with their experiences with traditional medicine. Thus, most of them believe that there is some value in traditional medicine. Those who assert the value of traditional medicine base their beliefs upon empirical evidence; i.e. people they know who have been cured by traditional specialists. However, regardless of their ideological position, all of the nurses believe that there is a strong connection between traditional specialists and sorcery. As a result the nurses are either against cooperation with traditional specialists or they believe that use of a traditional specialist is a patient's private affair. In their vicw cooperation means that traditional specialists should refer patients to biomedicine not the reverse.

For example when asked whether they would refer patients to traditional specialists some of the nurses' comments were:

Traditional healers know nothing about the virus. The only collaboration that is necessary is to know that they (traditional healers) exist on their own. not attached to hospitals or clinics, that they are free to refer what they can't cure to the scientific sector.

\section{Another said:}

"I have always believed in the scientific sector".

Another felt that for patients to go to traditional specialists was a 'waste of time'. She went on to say:

If the people would come straight to hospitals when ill they'd get cured. Going to the traditional specialist and then coming to the hospital only makes the illness advance unchecked.

This informant further said that she believed that most traditional specialists were 'tricksters'. Another nurse said that she was unwilling to work with traditional specialists because they use poisons on people'.

Socialized in both traditional and biomedical beliefs about health and illness, these nurses are ambivalent about cooperation between traditional specialists and biomedicine. Bailey [21] characterized brokers as persons who profit materially from their position. In Botswana the nurses ambivalence about cooperation with traditional specialists suggests that brokers do not always profit materially in their role. For them, the broker role creates a dialectical tension that results in ambivalence. The nurses do not wish to cooperate with spiritual healers because they view them as being more 'religious' than health careproviding.

In response to whether they would refer patients to spiritual healers, most of the nurses said that they do not believe in spiritual healers and therefore are not willing to collaborate with them. The nurses' view of collaboration with spiritual healers is to allow the healer to come and pray for patients. The nurses have minimal knowledge about the practice of spiritual healing. In a sense, the nurses could be accused of separating religion from healing and thereby violating a baTswana tradition. The nurses' view of spiritual healers may be influenced by their social class. Nursing is considered a middle-class occupation in Botswana. Although the nurses identify themselves as 'Christians', they do not attend African Christian Independent churches. However, they do attend the churches that were originally established by the missionaries.

Those nurses who do not completely reject traditional specialists, do not seem to separate healing from religion. Several of these nurses stated, "They (traditional specialists) are a part of our religion". They recognized the traditional specialists as part of their religion, and accept that traditional specialists heal some people. However the pervasive belief in sorcery preciudes nurses from desiring to cooperate with traditional specialists.

\section{CONCLUSION}

Although a number of difficulties have been identified in regard to the incorporation of ethnomedical specialists into the biomedical system, there have been some successful programs [22]. The situation in Botswana provides some additional data in which to view cooperation between ethnomedical and biomedical systems. There is a need to not only identify the varieties of ethnomedical practitioners in a given situation of medical pluralism, but also to identify those biomedical practitioners whose positions are crucial to the success of any incorporation program. Although physicians in the United States may be in the position to thwart proposed health programs, it must not be assumed that physicians wield the same power in other countries. Once the biomedical practitioners in crucial positions have been identified, then their views of cooperation must be ascertained and their willingness to participate in an incorporation program secured.

In Botswana it is the nurses who occupy the crucial position vis a vis cooperation. Any program that seeks incorporation between the biomedical and 
ethnomedical systems in Botswana requires their cooperation. Their current ambivalence about cooperation with traditional specialists and their refusal to cooperate with spiritual healers is based upon their knowledge of the former and their lack of knowledge about the latter.

\section{REFERENCES}

1. World Health Organization. Report of the International Conference on Primary Health Care. Geneva, 1978.

2. World Health Organization. The Alma-Alta conference of primary health care. Wld Hlth Org. 32, 431-438, 1978.

3. World Health Organization. The Promotion and Development of Traditional Medicine. WHO, Technical Report Series 62. WHO, Geneva, 1978.

4. Pillsbury B. L. K. Policy and evaluation perspectives on traditional health practitioners in national health care systems. Soc. Sci. Med. 16, 1825-1834, 1982.

5. Neumann A. K. and Lauro $P$. Ethnomedicine and biomedicine linking. Soc. Sci. Med. 16, 1817-1824, 1982.

6. Hughes C. C. Elkomedicine. International Encyclopedia of the Social Sciences, Vol. 10, pp. 87-92. The Free Press, New York, 1968.

7. Press I. Problems in the definition and classification of medical systems. Soc. Sci. Med. 14B, 45-57, 1980.

8. Yoder P. S. Biomedical and ethnomedical practice in rural Zaire. Soc. Sci. Med. 16, 1851-1858, 1982.
9. Schapera I. A Handbook of Tswana Law and Custom. 2nd edition. Oxford University Press for International African Institute, London, 1955.

10. Schapera I. The Ethnic Composition of Tswana Tribes. London School of Economics and Political Science. London, 1952.

11. Brink P. J. Traditional birth attendants among the Annang of Nigeria: current practices and proposed programs. Soc. Sci. Med. 16, 1883-1892. 1982.

12. Karujhe K. Personal communication, 1983.

13. Evans-Pritchare E. E. Witchcraft, Oracles and Magic Among the Azande. Claredon Press, Oxford, 1937.

14. Schapera I. Sorcery and witcheraft in Bechuanaland. Afr. Affairs 51, 41-50, 1952.

15. Barbee E. L. Health action and conflict among professional health care providers in Botswana. Unpublished Ph.D. dissertation, University of Washington, Seattle, 1979.

16. Maclean C. M. U. Hospitals or healers? An attitude survey in Ibadan. Hum. Org. 25, 131-139, 1966.

17. Janzen J. The Quest for Therapy in Lower Zaire. University of California Press, Berkeley, 1978.

18. Twumasi P. A. Medical Systems in Ghana: Study in Medical Sociology. Ghana Publishing Corp., Accra, 1975.

19. Wolfe E. R. Aspects of group relations in a complex society: Mexico. Am. Anthrop. 58, 1065-1078, 1956.

20. Comaroff J. L. Healing and cultural transformationthe Tswana of Southern Africa. Soc. Sci. Med. 15B, 267-278.

21. Bailey F. G. Strategem and Spoils. Schocken Books, New York, 1969.

22. See for example the special issue of Soc. Sci. Med. Utilization of indigenous healers in national health systems. Vol. 16, No. 21, 1982. 